\title{
Quantitative trait loci markers derived from whole genome sequence data increases the reliability of genomic prediction
}

\author{
R. F. Brøndum, ${ }^{* 1}$ G. Su, ${ }^{*}$ L. Janss, ${ }^{*}$ G. Sahana, ${ }^{*}$ B. Guldbrandtsen, ${ }^{*}$ D. Boichard,† and M. S. Lund ${ }^{*}$ \\ ${ }^{*}$ Center for Quantitative Genetics and Genomics, Department of Molecular Biology and Genetics, Blichers Allé 20, Aarhus University, \\ DK-8830 Tjele, Denmark \\ †Institut National de la Recherche Agronomique (INRA), UMR 1313 Génétique Animale et Biologie Intégrative, 78350 Jouy-en-Josas, France
}

\begin{abstract}
This study investigated the effect on the reliability of genomic prediction when a small number of significant variants from single marker analysis based on whole genome sequence data were added to the regular $54 \mathrm{k}$ single nucleotide polymorphism (SNP) array data. The extra markers were selected with the aim of augmenting the custom low-density Illumina BovineLD SNP chip (San Diego, CA) used in the Nordic countries. The single-marker analysis was done breed-wise on all 16 index traits included in the breeding goals for Nordic Holstein, Danish Jersey, and Nordic Red cattle plus the total merit index itself. Depending on the trait's economic weight, 15, 10, or 5 quantitative trait loci (QTL) were selected per trait per breed and 3 to 5 markers were selected to tag each QTL. After removing duplicate markers (same marker selected for more than one trait or breed) and filtering for high pairwise linkage disequilibrium and assaying performance on the array, a total of 1,623 QTL markers were selected for inclusion on the custom chip. Genomic prediction analyses were performed for Nordic and French Holstein and Nordic Red animals using either a genomic BLUP or a Bayesian variable selection model. When using the genomic BLUP model including the QTL markers in the analysis, reliability was increased by up to 4 percentage points for production traits in Nordic Holstein animals, up to 3 percentage points for Nordic Reds, and up to 5 percentage points for French Holstein. Smaller gains of up to 1 percentage point was observed for mastitis, but only a 0.5 percentage point increase was seen for fertility. When using a Bayesian model accuracies were generally higher with only $54 \mathrm{k}$ data compared with the genomic BLUP approach, but increases in reliability were relatively smaller when QTL markers were included. Results from this study indicate that the reliability of genomic prediction can be increased by including
\end{abstract}

Received October 22, 2014.

Accepted March 12, 2015.

${ }^{1}$ Corresponding author: rasmusf.brondum@mbg.au.dk markers significant in genome-wide association studies on whole genome sequence data alongside the $54 \mathrm{k}$ SNP set.

Key words: custom chip, genomic prediction, quantitative trait loci, Bos taurus

\section{INTRODUCTION}

The accuracy of genomic prediction is highly dependent on the linkage disequilibrium (LD) between the genotyped markers and actual causative variants (de Roos et al., 2008; de Los Campos et al., 2013). In dairy cattle, genomic predictions are usually done using the Illumina Bovine SNP50 (54k) SNP chip (Illumina Inc., San Diego, CA), where the distance between the markers might dictate a low level of LD. Increasing the marker density to $777 \mathrm{k}$ or high density (HD) has only increased the reliability by around 1 percentage point (VanRaden et al., 2011; Erbe et al., 2012; Su et al., 2012). In a study on simulated whole genome sequence data, it was shown that even at an already high marker density a further inclusion of causative variants led to a higher accuracy of genomic prediction (Meuwissen and Goddard, 2010), but the selection of markers included on the commercial chips is without reference to their association with phenotypes in dairy cattle. With the advent of next-generation sequencing (NGS) technologies, whole-genome sequence data has become available at a reasonable price. This makes it possible to sequence substantial numbers of bulls, which can be used as a reference to impute animals already genotyped with $54 \mathrm{k}$ or HD chips. The NGS data from the 1,000 Bull Genomes project (Daetwyler et al., 2014) have dramatically increased the number of animals available as a reference for imputation, such that common sequence markers (minor allele frequency $>0.1$ ) can now be imputed with an accuracy of more than 0.8 (Brøndum et al., 2014; van Binsbergen et al., 2014). However, with current genomic prediction models and computational resources, the sizes of data sets with imputed sequence are too large for all markers to be included in the model at the same time. Conversely, 
genome-wide association studies (GWAS) based on sequence data have shown higher power to identify putative causative variants and shows stronger signals of association (Daetwyler et al., 2014; Höglund et al., 2014; Sahana et al., 2014a). By using GWAS to identify QTL and including these in genomic prediction models, it might be possible to increase the accuracy of genomic prediction (Van den Berg et al., 2014).

In Nordic countries, cows are genotyped on a large scale using the Illumina BovineLD chip (7k). This chip has been designed for imputation performance such that $54 \mathrm{k}$ markers can be imputed with high accuracy (Boichard et al., 2012). This chip can be augmented by additional markers, for example, better imputation by increasing the overlap with the $54 \mathrm{k}$ chip or with markers that are expected to increase the accuracy of genomic prediction. These markers might then be imputed with higher accuracy than from next generation sequence data into existing $54 \mathrm{k}$ data. In the current study, we investigated the effect on the accuracy of genomic prediction when markers strongly associated with QTL in GWAS with whole-genome sequence data are included in the prediction models.

\section{MATERIALS AND METHODS}

\section{GWAS}

Data. For GWAS, phenotypic data were available as EBV for the 16 index traits included in the Nordic total merit index as well as the index itself; all traits are listed in Table 1 and Table 2. Data were available for the 3 major Nordic dairy breeds and comprised approximately (varies slightly for different traits) 5,000 Nordic Holstein bulls, 1,100 Danish Jersey bulls, and 4,500 Nordic Red bulls. All bulls were imputed to fullsequence data using a 2 -step approach, where $54 \mathrm{k}$ data were initially imputed to HD data and subsequently to the full sequence level. For more detail on this imputation, see Höglund et al. (2014).

Association Analysis. Genome-wide association analysis was carried out separately for the 3 breeds. The analysis was done using a linear mixed model approach where sires were fitted as a random variable that only considered sire-son relationships (for details see Sahana et al., 2014a). The sequence variants were filtered based on their imputation accuracy $\left(\mathrm{AR}^{2} \geq 0.95\right.$, where $\mathrm{AR}^{2}$ is the square of the estimated correlation between the imputed genotype with highest posterior probability with the true genotype of the marker; Browning and Browning, 2009). Larger values of $\mathrm{AR}^{2}$ indicate more accurate genotype imputation. The total number of markers [SNP or INDELS (insertion-deletions)] analyzed was $\sim 10$ million on 29 autosomes.
Table 1. Distribution of traits in categories based on the economic value of the trait ${ }^{1}$

\begin{tabular}{lll}
\hline Category I & Category II & Category III \\
\hline Mastitis & Longevity & NTM $^{2}$ \\
Fertility & Other diseases & Growth \\
Legs and Feet & Birth & Udder conformation \\
Milk yield & Calving & Body-conformation \\
Fat yield & & Milking-speed \\
Protein yield & & Yield \\
& & Temperament \\
\hline
\end{tabular}

${ }^{1}$ The number of QTL selected per trait was 15,10 , or 5 for category I, II, and III, respectively.

${ }^{2}$ Nordic total merit index.

Marker Selection. The 17 indices were loosely classified into 3 categories based on their relative economic importance (see Table 1). The QTL were ranked within breed based on the strength of the association signal and visual assessment of the QTL peak. The numbers of QTL selected were at most 15, 10, and 5 for the 3 categories for each breed (only if there was a sufficient number of genome-wide significant QTL segregating within the breed). Three to 5 associated markers were initially selected to cover each QTL. This was done manually for each QTL. The selection criteria were $P$-values, functional annotation (such as missense variants), and representation for multiple peaks within a QTL region and distance between markers, so if 2 consecutive markers showed similar association strength only the one with highest $\log _{10}(P$-value $)$ was included. All markers selected for a breed for all the traits, as described previously, were collected and the duplications were removed (if the same marker was selected for 2 traits). The markers were then pruned within

Table 2. Number of QTL per trait and breed covered by $\geq 1$ SNP in the linkage disequilibrium chip

\begin{tabular}{|c|c|c|c|}
\hline Indices & Holstein & $\begin{array}{c}\text { Nordic } \\
\text { Red }\end{array}$ & Jersey \\
\hline Birth & 10 & 15 & 10 \\
\hline Body confirmation & 6 & 8 & 5 \\
\hline Calving & 13 & 12 & 15 \\
\hline Fat yield & 15 & 14 & 14 \\
\hline Fertility & 19 & 15 & 15 \\
\hline Growth & 8 & 9 & 5 \\
\hline Legs and feet & 17 & 18 & 10 \\
\hline Longevity & 8 & 11 & 14 \\
\hline Mastitis & 16 & 17 & 25 \\
\hline Milk yield & 13 & 18 & 16 \\
\hline Milking speed & 5 & 7 & 5 \\
\hline NTM index ${ }^{1}$ & 6 & 8 & 9 \\
\hline Other diseases & 23 & 21 & 13 \\
\hline Protein yield & 22 & 10 & 14 \\
\hline Temperament & 6 & 7 & 0 \\
\hline Udder conformation & 8 & 8 & 8 \\
\hline Yield index & 16 & 8 & 13 \\
\hline
\end{tabular}

${ }^{1}$ Nordic total merit index. 
Table 3. Number of animals and mean reliability of deregressed proofs $\left(\mathrm{R}_{\mathrm{DRP}}^{2}\right)$ in the training and validation population

\begin{tabular}{|c|c|c|c|c|c|c|}
\hline \multirow[b]{2}{*}{ Population } & \multirow[b]{2}{*}{ Split } & \multirow[b]{2}{*}{ Trait } & \multicolumn{2}{|c|}{ Training } & \multicolumn{2}{|c|}{ Validation } \\
\hline & & & $\mathrm{N}$ & $\mathrm{R}_{\mathrm{DRP}}^{2}$ & $\mathrm{~N}$ & $\mathrm{R}_{\mathrm{DRP}}^{2}$ \\
\hline \multirow[t]{3}{*}{ Nordic Holstein } & \multirow[t]{3}{*}{$2005 / 01 / 01$} & Milk, fat, protein & 3,949 & 0.94 & 1,046 & 0.94 \\
\hline & & Fertility & 3,947 & 0.69 & 1,043 & 0.68 \\
\hline & & Mastitis & 3,951 & 0.83 & 1,046 & 0.82 \\
\hline \multirow[t]{2}{*}{ French Holstein } & \multirow[t]{2}{*}{$2005 / 01 / 01$} & Milk, fat, protein & 3,804 & 0.76 & 1,369 & 0.74 \\
\hline & & Mastitis & 2,988 & 0.32 & 1,194 & 0.23 \\
\hline \multirow[t]{3}{*}{ Nordic Red } & \multirow[t]{3}{*}{$2005 / 01 / 01$} & Milk, fat, protein & 3,570 & 0.96 & 874 & 0.93 \\
\hline & & Fertility & 3,605 & 0.79 & 870 & 0.67 \\
\hline & & Mastitis & 3,570 & 0.85 & 874 & 0.75 \\
\hline
\end{tabular}

breed using the software PLINK v1.07 (http://pngu. mgh.harvard.edu/purcell/plink/) with a threshold for the pair-wise LD of $r^{2} \geq 0.95$, where $r^{2}$ is the squared correlation. Finally, all the selected markers from the 3 breeds were combined and duplications were removed (if the same marker was selected for 2 breeds). As the aim of this marker selection was to augment them onto a custom Illumina BovineLD chip, the markers were run through the Illumina Assay Design Tool (http:// support.illumina.com/array/array_software/assay_design_tool.html) to assess how well the markers would perform in the assay. Markers that were predicted to perform poorly in the assay were exchanged with the other strongly associated SNP for the same trait and breed based on the criteria described above. The final set included a total of 1,623 markers selected across the 3 breeds and 17 indices. The number of QTL per trait and breed covered by 1 or more markers on the panel are shown in Table 2. As some markers showed significant association for more than 1 trait, the number of QTL per trait in some cases exceeded 15.

\section{Genomic Prediction}

Phenotypic Data. For the genomic prediction analysis, 3 different data sets were used. The first 2 were Nordic Holstein and Nordic Red (RDC), with 4,999 and 4,495 bulls, respectively. As all of the available phenotyped and genotyped Nordic bulls, including the validation populations for this analysis, had been used for the marker selection step, any gain in accuracy from the added QTL markers may be overestimated due to the indirect use of phenotypic information from the validation animals. For this reason, an additional data set consisting of 5,207 French Holstein bulls from the EuroGenomics collaboration (Lund et al., 2011) was chosen for a more independent validation. All data sets were split into reference and validation populations according to their date of birth. For an overview of reference and validation populations, see Table 3 . For all animals, phenotypes were available as deregressed breeding values (DRP) on the Nordic scale. The traits selected for this analysis were milk, fat and protein yield, fertility, and mastitis. As fertility is defined differently in the Nordic countries and France, conversion to the Nordic scale results in a very low reliability of the breeding values; hence this trait was left out for the French Holsteins. Furthermore, French DRP for mastitis is based only on mastitis registrations, whereas Nordic values have SCC included as a correlated trait. This results in a low reliability of mastitis for the French animals in Nordic scale.

Genotypic Data. All bulls had Illumina 54k genotypes version 1 or 2 . The Nordic bulls were imputed to full genome sequence data using a 2-step approach. Animals were first imputed to HD level using a multibreed reference of 3,383 animals that had been genotyped with the Illumina BovineHD chip. These imputed genotypes were subsequently imputed to the whole genome sequence level using a multi-breed reference of 1,228 animals from run 4 of the 1,000 Bull Genomes project (Daetwyler et al., 2014) and private data from Aarhus University. Data sets at different densities were prephased with BEAGLE4 r1274 (Browning and Browning, 2013) and imputations were done using IMPUTE2 v2.3.1 (Howie et al., 2011), as suggested by Brøndum et al. (2014). Genotypes for the QTL markers were then extracted from the whole genome sequence data. After quality filtering and removing markers that overlapped with the $54 \mathrm{k}$ data, 1,443 QTL markers were available for analysis. For the French Holsteins, QTL markers were obtained by using the Nordic Holsteins with 54k data and the added imputed QTL markers as a reference for imputation. Imputations for the French Holstein bulls were done using Beagle v3.3.2 (Browning and Browning, 2009). For all data sets, markers with minor allele frequency less than 0.01 were removed.

Prediction Models. Analyses comprised 3 marker panel scenarios: (1) only the $54 \mathrm{k}$ data, (2) $54 \mathrm{k}$ data combined with the imputed QTL markers, and (3) the $54 \mathrm{k}$ data and the imputed QTL markers as separate marker sets with their own variance components. Three 
sets of QTL markers were used in genomic prediction: (I) all 1,443 QTL markers were included for all traits, (II) only QTL markers selected for the specific trait were used, and (III) only QTL markers selected for the specific trait and breed were used. The data were analyzed using a genomic BLUP (GBLUP) model and a Bayesian mixture model that included either 1 or 2 random genetic effects.

(1) GBLUP models: The model with 1 component was

$$
\mathbf{y}=\mathbf{1} \mu+\mathbf{Z a}+\mathbf{e},
$$

and the model with 2 random genetic effects was

$$
\mathbf{y}=1 \mu+\mathbf{Z}_{54 \mathrm{k}} \mathbf{a}_{54 k}+\mathbf{Z}_{\mathrm{QTL}} \mathbf{a}_{\mathrm{QTL}}+\mathbf{e}
$$

where $\mu$ was the overall mean; $\mathbf{a}, \mathbf{a}_{54 \mathrm{k}}$, and $\mathbf{a}_{\mathrm{QTL}}$ were the vectors of additive genetic values; e was the vector of residual errors; $\mathbf{Z}$ was an incidence matrix relating additive genetic values for the $54 \mathrm{k}$ data or the combined $54 \mathrm{k}$ and QTL data to phenotypes; and $\mathbf{Z}_{54 \mathrm{k}}$ and $\mathbf{Z}_{\mathrm{QTL}}$ were incidence matrices for the $54 \mathrm{k}$ data and the QTL data. It was assumed that $\mathbf{a}_{\mathrm{i}} \sim N\left(0, \mathbf{G}_{\mathrm{i}} \sigma_{\mathrm{gi}}^{2}\right)$, where the genomic relationship matrix $\mathbf{G}_{\mathrm{i}}$ was constructed according to method 1 in (VanRaden, 2008) based on the $54 \mathrm{k}$ data, the QTL marker data, or the combined marker data, and $\sigma_{g i}^{2}$ is the additive genetic variance explained by the markers in $\mathbf{G}_{\mathrm{i}}$. Heterogeneous residual variances of DRP were accounted for by dividing residual variance by a weight derived from the reliability of DRP $\left(\mathbf{R}_{\text {DRP }}^{2}\right)$ given by $\mathrm{R}_{\mathrm{DRP}}^{2} /\left(1-\mathrm{R}_{\mathrm{DRP}}^{2}\right)$.

(2) Bayesian 2-distribution mixture models: The first was given by

$$
\mathbf{y}=\mathbf{1} \mu+\mathbf{X g}+\mathbf{e},
$$

and the second by

$$
\mathbf{y}=\mathbf{1} \mu+\mathbf{X}_{54 \mathrm{k}} \mathbf{g}_{54 \mathrm{k}}+\mathbf{X}_{\mathrm{QTL}} \mathbf{g}_{\mathrm{QTL}}+\mathbf{e},
$$

where $\mu$ and $\mathbf{e}$ were defined as before; $\mathbf{g}, \mathbf{g}_{54 \mathrm{k}}$, $\mathrm{g}_{\mathrm{QTL}}$ were vectors of effects for the corresponding set of markers; $\mathbf{X}$ is a genotype matrix for the $54 \mathrm{k}$ data or combined $54 \mathrm{k}$ and QTL data; and $\mathbf{X}_{54 \mathrm{k}}$ and $\mathbf{X}_{\mathrm{QTL}}$ are genotype matrices for respectively 54k and QTL markers. Prior distributions for SNP effects are given as $\mathbf{g} \sim \pi N\left(0, \mathbf{I} \sigma_{1}^{2}\right)+(1-\pi) N\left(0, \mathbf{I} \sigma_{2}^{2}\right)$, where $\mathbf{I}$ is an identity matrix. In this study, $\sigma_{1}^{2}$ was set to $10^{-4}$, whereas the variance in the second distribution and the mixing parameter are estimated from the data with priors $\sigma_{2}^{2} \propto 1$ and $\pi \sim \beta(10,1)$. For the 2 component model separate $\pi$ and $\sigma_{2}^{2}$ were used for the $54 \mathrm{k}$ and QTL markers. Predicted breeding values were obtained as posterior means from a Markov chain Monte Carlo sampler run for 50,000 iterations where the first 10,000 were discarded as burn-in. Convergence and mixing was validated by correlating direct genomic values from repeated runs in the Nordic Holsteins, which were greater than 0.999 for all traits and scenarios.

Analysis for the GBLUP model was done using DMU (Madsen and Jensen, 2013) and analysis for the Bayesian model was done using Bayz (Kapell et al., 2012). For all models, the reliability of direct genomic values (DGV) was calculated as the squared correlation of DGV and DRP divided by the mean reliability of DRP in the test population, and the bias was calculated as the regression of DRP on DGV.

\section{RESULTS}

Comparing Bayesian analyses with the entire QTL set, trait-specific QTL, or trait- and breed-specific QTL (Appendix Tables A1-A3), the highest reliabilities of genomic prediction were found using the entire QTL set. This was true irrespective of whether the QTL markers were pooled with the $54 \mathrm{k}$ markers or treated as a separate component. The only exception from this was for mastitis in French Holsteins, where a minor advantage of 0.3 percentage points in reliability was seen for trait- and breed-specific markers compared with using the whole set in the Bayesian model with separate components. For the GBLUP model (Appendix Tables A1-A3) the entire QTL set also gave the highest reliabilities when combining the QTL markers with the $54 \mathrm{k}$ data for all 3 breeds. However, for French Holstein and Nordic Red animals, 0.6 to $1.5 \%$ higher reliabilities were found for production traits when using only the trait- or trait- and breed-specific QTL markers as a separate component.

The rest of the results are shown only for the scenario with the entire QTL marker set, as this scenario yielded the highest reliabilities overall. Reliabilities of DGV for all traits, test populations, and prediction models are shown in the left hand side of Figure 1. For the Nordic Holsteins, gains in reliability between 1.5 and 4 percentage points were seen for the production traits. Fertility did not seem to benefit from the added QTL markers, whereas a gain of 1.5 percentage points was 
seen for mastitis. When the different scenarios are compared, a clear trend toward an increase in reliability for production traits was observed when the QTL markers were combined with the 54k data. A larger increase was observed when the QTL and non-QTL markers were assigned separate variance components.

For the RDC bulls, gains in reliability between 0.7 and 2.9 percentage points were seen for production traits, whereas only 1 percentage point gain was seen for fertility. For mastitis, a small gain of 0.6 or 0.9 percentage points in reliability was observed when $54 \mathrm{k}$ and QTL markers were combined and a GBLUP or Bayesian model, respectively, was used. Similar to the
Nordic Holsteins, increases in reliability for traits other than mastitis seemed to follow the emphasis put on the QTL markers (i.e., larger gains were seen for the separate variance component setup than when combining the markers).

For the French Holsteins, gains in reliability between 2 and 5 percentage points were observed for the production traits. For mastitis we only saw a smaller increase of up to 0.9 percentage points. For production traits we again observed a trend toward a larger increase in the reliability when the QTL markers were assigned as a separate variance component. An exception here was fat yield using the Bayesian model, where the high-
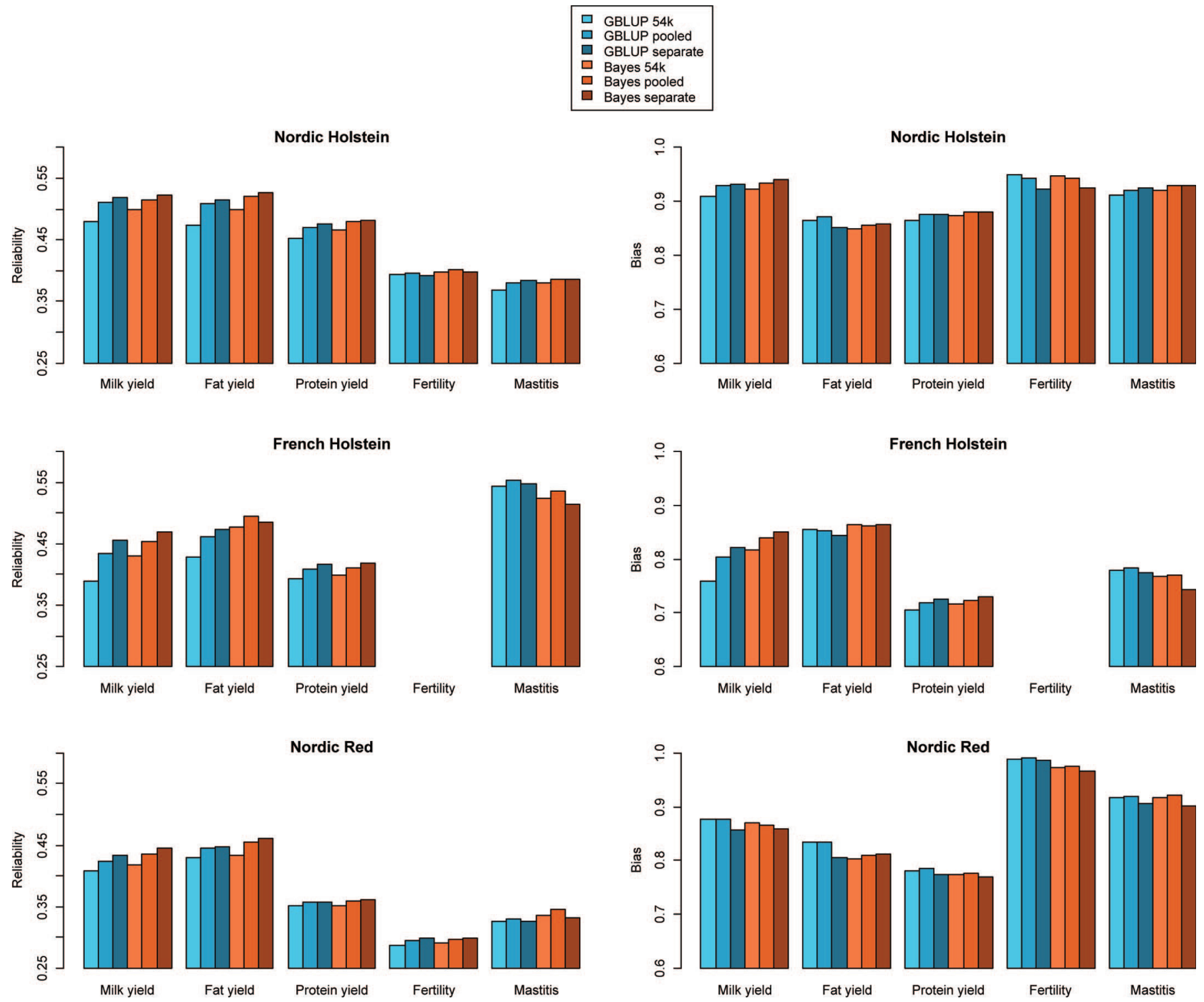

Nordic Red
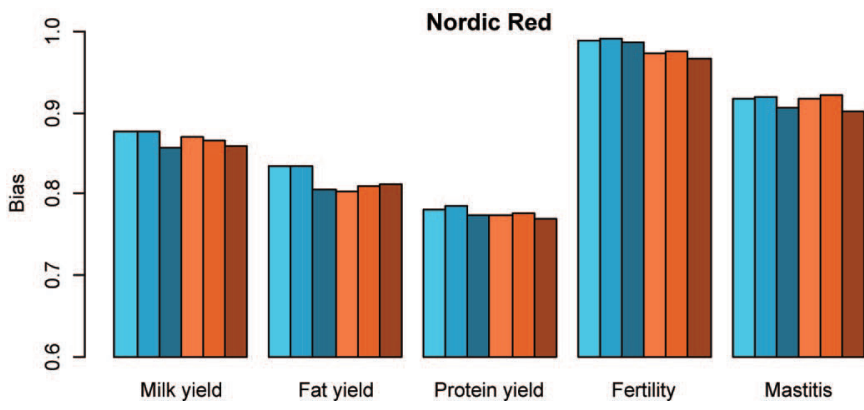

Figure 1. Reliability and bias of direct genomic values. Bars within breed or trait, going from left to right, are a genomic BLUP (GBLUP) model with 54k data, 54k data pooled with QTL markers, or 54k data and QTL markers treated as separate components, and a Bayesian 2-mixture model on 54k data, 54k data pooled with QTL markers, or 54k data and QTL markers treated as separate components. Color version available online. 


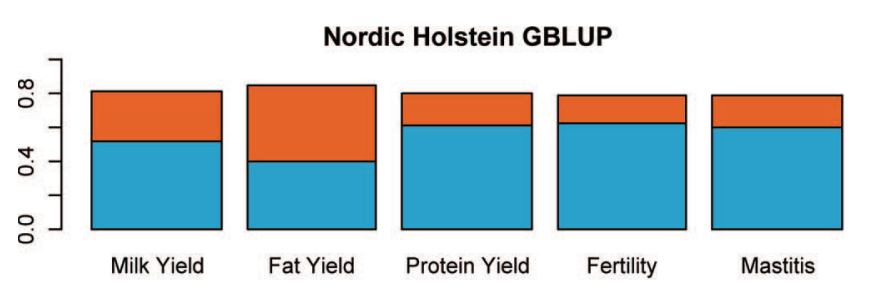

French Holstein GBLUP
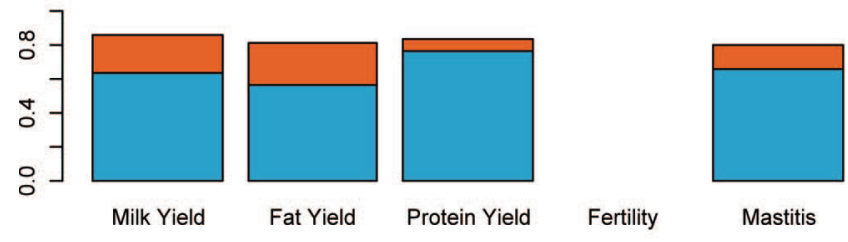

Nordic Red GBLUP
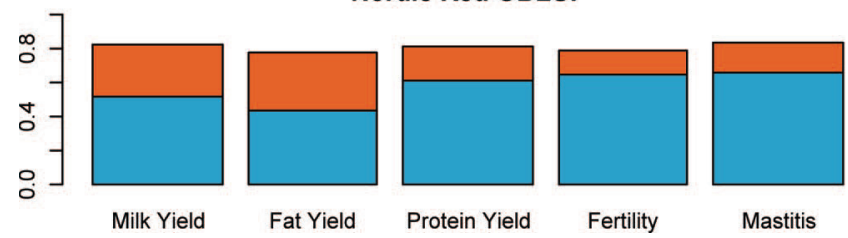
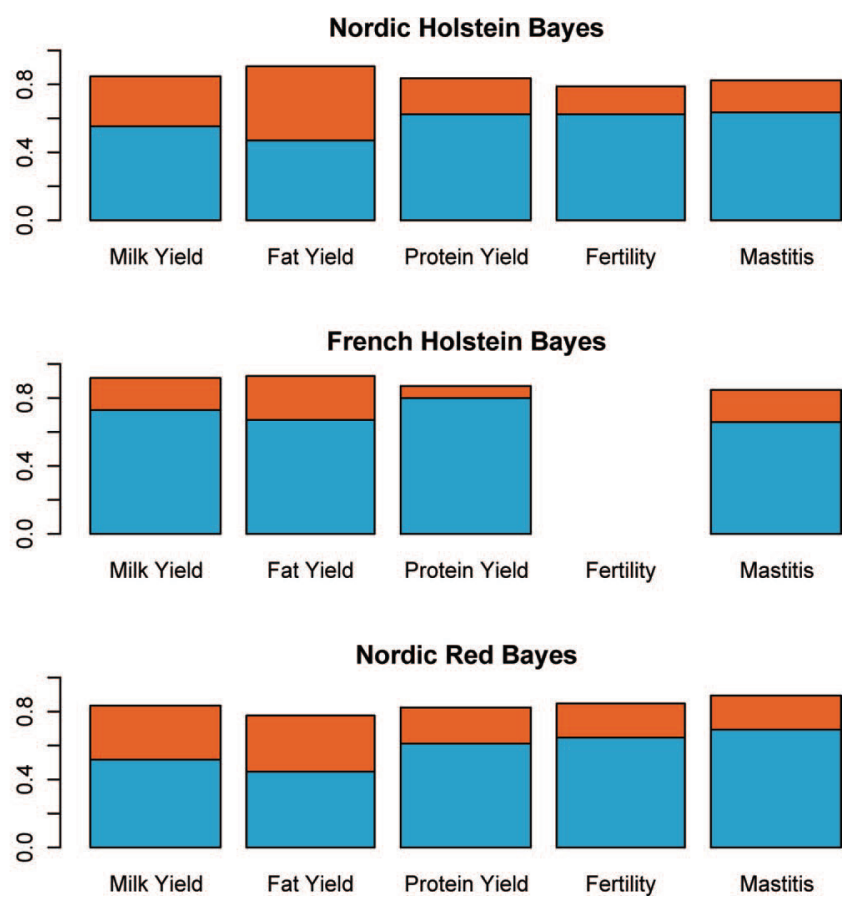

Figure 2. Proportion of total direct genomic value (DGV) variance explained by partial DGV for either 54k (light gray/blue) or QTL (dark gray/orange) data for validation animals in the scenarios with separate variance components. Total DGV variance is calculated as the variance of the sum of the 2 partial DGV. GBLUP = genomic BLUP. Color version available online.

est accuracy was found in the combined scenario. For fat yield in the French Holsteins, the Bayesian model with separate variance components did not converge properly, with the tendency that the $\pi$ parameter for the QTL markers would move toward 1, whereas the variance on the SNP effects in the second distribution for the QTL markers moved toward infinity. To counteract this we fixed the $\pi$ parameter to the posterior value from the Nordic Holstein for this scenario for the French Holsteins. The high reliabilities observed for mastitis is caused by the low reliabilities of DRP in the Nordic scale (see Table 3).

Bias of DGV is shown in the right-hand side of Figure 1. An inflation of the DGV was observed for most traits and populations with regression coefficients between 0.71 and 0.99 , where the value closest to 1 was observed for fertility in the RDC population. Regression coefficients varied by up to $9 \%$ within trait or population, but no clear pattern across the scenarios was observed.

Figure 2 shows the proportion of the total DGV variance of partial DGV for the 54k markers or the QTL markers as well as the total DGV in the scenario with separate variance components for the 2 marker sets. In general, the proportion of variance explained by the QTL markers was large compared with the $54 \mathrm{k}$ data when considering the much lower number of markers in the QTL set. Furthermore, the variances of the full
DGV were larger than the sum of variances for the 2 components, showing a positive covariance.

\section{DISCUSSION}

Gains in reliability when including the QTL markers are higher than what has previously been reported when going from $54 \mathrm{k}$ data to HD despite a much smaller number of markers being added. In a study of the Nordic Holstein population an increase in the reliability of DGV of 0.5 percentage points for protein was reported when extending the SNP set from $54 \mathrm{k}$ to HD data (Su et al., 2012), whereas results here show an increase of 1.7 percentage points when simply combining the markers and up to 2.2 percentage points when the QTL markers are assigned as a separate variance component. Two possible reasons could explain these differences. First, the extra markers included in our study are obtained from whole-genome sequence data and are likely closer to actual causal mutations. Second, when going from $54 \mathrm{k}$ data to HD a lot of extra markers which are not expected to have an effect on traits of interest are also included; this might add extra noise in the genotype data. Both points are supported by results from Van den Berg et al. (2014), where it was shown by simulating QTL in actual sequence data that a genomic relationship constructed from HD markers had a lower 
correlation with the causal relationship than one constructed from markers close to causal variants.

As validation bulls in our study were included in the GWAS which the marker selection was based on, the effect of adding the markers might be overestimated in the Nordic Holstein and RDC validation bulls. However, results from French Holstein showed gains of the same magnitude as the gains observed in Nordic Holstein when including the QTL markers in the genotype data. The observed reliabilities for production traits in French Holstein were lower than for the Nordic Holsteins, whereas previous studies have reported similar reliabilities for protein yield using the $54 \mathrm{k}$ data for Nordic and French Holsteins (Dassonneville et al., 2011; Lund et al., 2011). However, in these previous studies both populations used phenotypes on their own national scale, whereas DRP in our study had a lower reliability for both training and validation animals in the French Holstein, as they had been converted to the Nordic scale. This could cause the lower level of reliability of DGV for the French animals.

Larger gains in the reliability of DGV were seen when QTL markers were treated as a separate variance component instead of just being combined with the $54 \mathrm{k}$ data, and smaller gains were found for the Bayesian model than for the GBLUP. This is likely because a GBLUP model with 2 components of genetic effects allows effects of QTL markers to have larger variance than those of the $54 \mathrm{k}$ markers, thus putting more weight on the QTL markers. The Bayesian model had the ability to identify markers in close LD with QTL even when only $54 \mathrm{k}$ data were used. Thus, a relatively smaller gain from the QTL markers was noted when using the Bayesian model. Accordingly, the realized reliabilities from the GBLUP model were closer to those obtained from the Bayesian model (i.e., for Nordic Holstein when only the $54 \mathrm{k}$ data were used the average reliability over the 5 traits was 0.434 and 0.457 for GBLUP and the Bayesian method, respectively, whereas inclusion of the QTL markers with a separate variance components increased the average reliabilities to 0.457 and 0.463 , decreasing the difference by 1.7 percentage points).

The largest gains in reliability were found for production traits, especially for milk and fat yield, for which some major QTL have been reported (e.g., Grisart et al., 2002; Wang et al., 2012). Sahana et al., (2014b) also reported that the top 10 QTL for fat and milk yield explained 23.7 and $22.6 \%$ of the additive genetic variance, compared with only 14.8 and $10.9 \%$ for fertility and protein yield, respectively, in Nordic Holstein. As the QTL markers were identified by within-breed analysis, this larger gain might be caused by QTL for production traits being more similar across breeds, possibly because production traits are more uniformly defined. Similarly the smaller gains for mastitis could be because of different QTL acting on the trait in the 3 breeds used for the marker selection. It has previously been shown that some of the major QTL for mastitis in Holstein are not segregating in Jersey. A QTL for mastitis on chromosome 6 , which explained about $4 \%$ of the additive genetic variance in Holstein, did not segregate in Jersey (Sahana et al., 2014a). Similarly 2 other major QTL for mastitis on BTA13 and BTA19 were not confirmed in Danish Jersey (Sahana et al., 2014a). It should, however, be noted that there was more power to identify QTL segregating in Holstein compared with Jersey due to the large difference in number of individuals with records $(5,000$ vs. 1,100$)$.

Using only trait- or trait- and breed-specific QTL markers instead of the entire QTL set had no effect when using the Bayesian model. This is reasonable, as the variable selection model ideally only chooses markers that have an effect on the trait in question, and thus should not suffer from including extra markers selected for other traits. The highest reliability seen for the Bayesian model when using QTL markers selected for a wide range of traits and breeds indicates that some of these QTL could have pleiotropic effects, and that markers selected for the same trait in a different breed might still have an effect, even if not a genome-wide significant one. For the GBLUP model with 2 genetic components, using only trait- or trait- and breed-specific QTL markers in some cases led to higher reliabilities in Nordic Red and French Holsteins compared with using the entire QTL marker set. As the GBLUP model does not choose which markers to use, this could be because the added markers selected from other traits or breeds only add noise and dilute the relationship described by the QTL markers chosen for the same trait and breed. However, for the Nordic Holsteins, the highest reliabilities were found when using the entire QTL marker set, even when using 2 genetic components.

Practical implementation of these QTL markers in genomic prediction is straightforward. If cows are routinely genotyped with the custom low-density chip including these markers, they could be made available for analysis by including them in the imputation procedures (i.e., when cows are imputed to $54 \mathrm{k}$ data, they could simultaneously work as a reference for imputing the QTL markers into the bulls). Results herein show that larger gains in reliability can be obtained if models are adjusted to allow for more emphasis on the QTL markers, but simply combining them with the $54 \mathrm{k}$ data also offers improvements. Similar results were found in a previous study by $\mathrm{Su}$ et al. (2014), which showed that weighting SNP used for building the g-matrix by posterior variances obtained from a Bayesian model led to increases in the reliability of genomic prediction. 
Possible further developments of the method suggested in the current study include considering alternative marker selection strategies with, for example, more trait-specific markers, using multiple breeds in GWAS, or using Bayesian mixture models for identification of QTL.

\section{CONCLUSIONS}

Results from our study show that including QTL markers, identified from sequence data by GWAS, in the marker panel used for genomic prediction can increase the reliability of direct genomic values. The largest gains were found for production traits and when the added QTL markers were treated as a separate variance component, which allows the model to put more emphasis on the known QTL.

\section{ACKKNOWLEDGEMENTS}

This work was supported by a grant 12-132452 (Genomic Selection in Animals and Plants, GenSAP) from the Danish Strategic Research Council and by funding from the INRA metaprogramme Selgen (Sélection Génomique). Nordic Cattle Genetic Evaluation (Aarhus, Denmark) and VikingGenetics (Randers, Denmark) are acknowledged for providing phenotypic and genotype data. The 1,000 bull genomes project (http:// www.1000bullgenomes.com) is kindly acknowledged for sharing data to impute the genome sequence.

\section{REFERENCES}

Boichard, D., H. Chung, R. Dassonneville, X. David, A. Eggen, S. Fritz, K. J. Gietzen, B. J. Hayes, C. T. Lawley, T. S. Sonstegard, C. P. Van Tassell, P. M. VanRaden, K. A. Viaud-Martinez, and G. R. Wiggans. 2012. Design of a bovine low-density SNP array optimized for imputation. PLoS ONE 7:e34130. http://dx.doi. org/10.1371/journal.pone.0034130.

Brøndum, R. F., B. Guldbrandtsen, G. Sahana, M. S. Lund, and G. Su. 2014. Strategies for imputation to whole genome sequence using a single or multi-breed reference population in cattle. BMC Genomics 15:728. http://dx.doi.org/10.1186/1471-2164-15-728.

Browning, B. L., and S. R. Browning. 2009. A unified approach to genotype imputation and haplotype-phase inference for large data sets of trios and unrelated individuals. Am. J. Hum. Genet. 84:210-223. http://dx.doi.org/10.1016/j.ajhg.2009.01.005.

Browning, B. L., and S. R. Browning. 2013. Improving the accuracy and efficiency of identity-by-descent detection in population data. Genetics 194:459-471. http://dx.doi.org/10.1534/ genetics.113.150029.

Daetwyler, H. D., A. Capitan, H. Pausch, P. Stothard, R. van Binsbergen, R. F. Brøndum, X. Liao, A. Djari, S. C. Rodriguez, C. Grohs, D. Esquerré, O. Bouchez, M.-N. Rossignol, C. Klopp, D. Rocha, S. Fritz, A. Eggen, P. J. Bowman, D. Coote, A. J. Chamberlain, C. Anderson, C. P. VanTassell, I. Hulsegge, M. E. Goddard, B. Guldbrandtsen, M. S. Lund, R. F. Veerkamp, D. A. Boichard, R. Fries, and B. J. Hayes. 2014. Whole-genome sequencing of 234 bulls facilitates mapping of monogenic and complex traits in cattle. Nat. Genet. 46:858-865. http://dx.doi.org/10.1038/ng.3034.
Dassonneville, R., R. F. Brøndum, T. Druet, S. Fritz, F. Guillaume, B. Guldbrandtsen, M. S. Lund, V. Ducrocq, and G. Su. 2011. Effect of imputing markers from a low-density chip on the reliability of genomic breeding values in Holstein populations. J. Dairy Sci. 94:3679-3686. http://dx.doi.org/10.3168/jds.2011-4299.

de Los Campos, G., A. I. Vazquez, R. Fernando, Y. C. Klimentidis, and D. Sorensen. 2013. Prediction of complex human traits using the genomic best linear unbiased predictor. PLoS Genet. 9:e1003608. http://dx.doi.org/10.1371/journal.pgen.1003608.

de Roos, A. P., B. J. Hayes, R. J. Spelman, and M. E. Goddard. 2008. Linkage disequilibrium and persistence of phase in Holstein-Friesian, Jersey and Angus cattle. Genetics 179:1503-1512. http:// dx.doi.org/10.1534/genetics.107.084301.

Erbe, M., B. J. Hayes, L. K. Matukumalli, S. Goswami, P. J. Bowman, C. M. Reich, B. A. Mason, and M. E. Goddard. 2012. Improving accuracy of genomic predictions within and between dairy cattle breeds with imputed high-density single nucleotide polymorphism panels. J. Dairy Sci. 95:4114-4129. http://dx.doi.org/10.3168/ jds.2011-5019.

Grisart, B., W. Coppieters, F. Farnir, L. Karim, C. Ford, P. Berzi, N. Cambisano, M. Mni, S. Reid, P. Simon, R. Spelman, M. Georges, and R. Snell. 2002. Positional candidate cloning of a QTL in dairy cattle: Identification of a missense mutation in the bovine DGAT1 gene with major effect on milk yield and composition. Genome Res. 12:222-231. http://dx.doi.org/10.1101/gr.224202.

Höglund, J. K., G. Sahana, R. F. Brøndum, B. Guldbrandtsen, B. Buitenhuis, and M. S. Lund. 2014. Fine mapping QTL for female fertility on BTA04 and BTA13 in dairy cattle using HD SNP and sequence data. BMC Genomics 15:790. http://dx.doi. org/10.1186/1471-2164-15-790.

Howie, B., J. Marchini, and M. Stephens. 2011. Genotype imputation with thousands of genomes. G3 (Bethesda) 1:457-470. http:// dx.doi.org/10.1534/g3.111.001198.

Kapell, D. N. R. G., D. Sorensen, G. Su, L. L. G. Janss, C. J. Ashworth, and R. Roehe. 2012. Efficiency of genomic selection using Bayesian multi-marker models for traits selected to reflect a wide range of heritabilities and frequencies of detected quantitative traits loci in mice. BMC Genet. 13:42. http://dx.doi. org/10.1186/1471-2156-13-42

Lund, M. S., A. P. W. De Roos, A. G. De Vries, T. Druet, V. Ducrocq, S. Fritz, F. Guillaume, B. Guldbrandtsen, Z. Liu, R. Reents, C. Schrooten, F. Seefried, and G. Su. 2011. A common reference population from four European Holstein populations increases reliability of genomic predictions. Genet. Sel. Evol. 43:43. http:// dx.doi.org/10.1186/1297-9686-43-43.

Madsen, P., and J. Jensen. 2013. A User's Guide to DMU. 1-32. http://dmu.agrsci.dk/DMU/Doc/Current/dmuv6_guide.5.2.pdf6.

Meuwissen, T., and M. Goddard. 2010. Accurate prediction of genetic values for complex traits by whole-genome resequencing. Genetics 185:623-631. http://dx.doi.org/10.1534/genetics.110.116590.

Sahana, G., B. Guldbrandtsen, B. Thomsen, L.-E. Holm, F. Panitz, R. F. Brøndum, C. Bendixen, and M. S. Lund. 2014a. Genomewide association study using high-density single nucleotide polymorphism arrays and whole-genome sequences for clinical mastitis traits in dairy cattle. J. Dairy Sci. 97:7258-7275. http://dx.doi. org/10.3168/jds.2014-8141.

Sahana, G., L. Janss, B. Guldbrandtsen, and M. S. Lund. 2014b. Genetic architecture of milk, fat, protein, mastitis and fertility studied using NGS data in Holstein cattle. Page 194 in Proc. 10th World Congr. Genet. Appl. Livest. Prod. Vancouver, Canada. http://www.wcgalp.com.

Su, G., R. F. Brøndum, P. Ma, B. Guldbrandtsen, G. P. Aamand, and M. S. Lund. 2012. Comparison of genomic predictions using medium-density $(\sim 54,000)$ and high-density $(\sim 777,000)$ single nucleotide polymorphism marker panels in Nordic Holstein and Red Dairy Cattle populations. J. Dairy Sci. 95:4657-4665. http:// dx.doi.org/10.3168/jds.2012-5379.

Su, G., O. F. Christensen, L. Janss, and M. S. Lund. 2014. Comparison of genomic predictions using genomic relationship matrices built with different weighting factors to account for locus-specific 
variances. J. Dairy Sci. 97:6547-6559. http://dx.doi.org/10.3168/ jds.2014-8210.

van Binsbergen, R., M. C. Bink, M. P. Calus, F. A. van Eeuwijk, B. J. Hayes, I. Hulsegge, and R. F. Veerkamp. 2014. Accuracy of imputation to whole-genome sequence data in Holstein Friesian cattle. Genet. Sel. Evol. 46:41. http://dx.doi.org/10.1186/12979686-46-41.

Van den Berg, I., B. Guldbrandtsen, C. Hoze, R. F. Brøndum, D. Boichard, and M. S. Lund. 2014. Across breed QTL detection and genomic prediction in French and Danish dairy cattle breeds. Page 490 in Proc. 10th World Congr. Genet. Appl. Livest. Prod. http:// www.wcgalp.com.
VanRaden, P. M. 2008. Efficient methods to compute genomic predictions. J. Dairy Sci. 91:4414-4423. http://dx.doi.org/10.3168/ jds.2007-0980.

VanRaden, P. M., J. R. O'Connell, G. R. Wiggans, and K. A. Weigel. 2011. Genomic evaluations with many more genotypes. Genet. Sel. Evol. 43:10. http://dx.doi.org/10.1186/1297-9686-43-10.

Wang, X., C. Wurmser, H. Pausch, S. Jung, F. Reinhardt, J. Tetens, G. Thaller, and R. Fries. 2012. Identification and dissection of four major QTL affecting milk fat content in the German Holstein-Friesian population. PLoS ONE 7:e40711. http://dx.doi. org/10.1371/journal.pone.0040711.

\section{APPENDIX}

Exact numbers for Figures 1 and 2 are given in Tables A1, A4, and A5. Reliabilities using only trait or trait and breed specific QTL markers are shown in Tables A2 and A3.

Table A1. Reliabilities of direct genomic values using a genomic BLUP (GBLUP) and a Bayesian 2-mixture model on 54k data, 54k data combined with QTL markers (54k_comb), or 54k data and QTL data treated as separate variance components (54k_qtl)

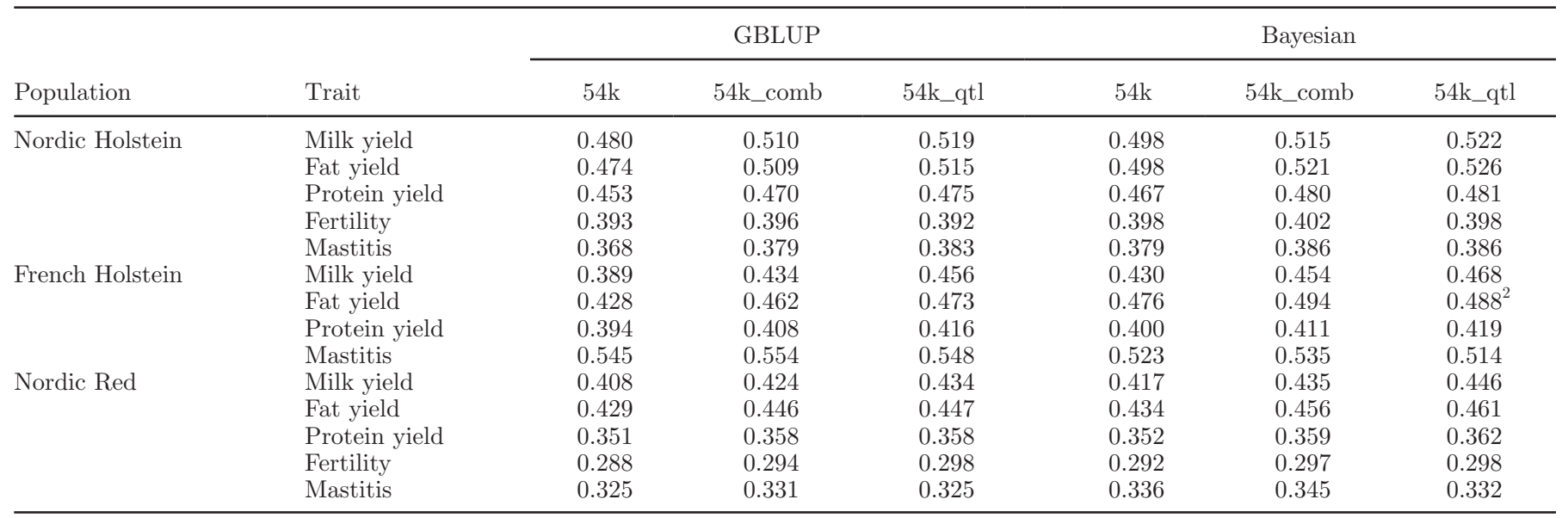

${ }^{1}$ Results in this table are obtained using the entire marker set from the linkage disequilibrium-chip as QTL markers.

${ }^{2}$ Fixed pi parameter from Nordic Holstein.

Table A2. Reliabilities of direct genomic values using a genomic BLUP (GBLUP) and a Bayesian 2-mixture model on 54k data, 54k data combined with QTL markers (54k_comb), or 54k data and QTL data treated as separate variance components (54k_qtl)

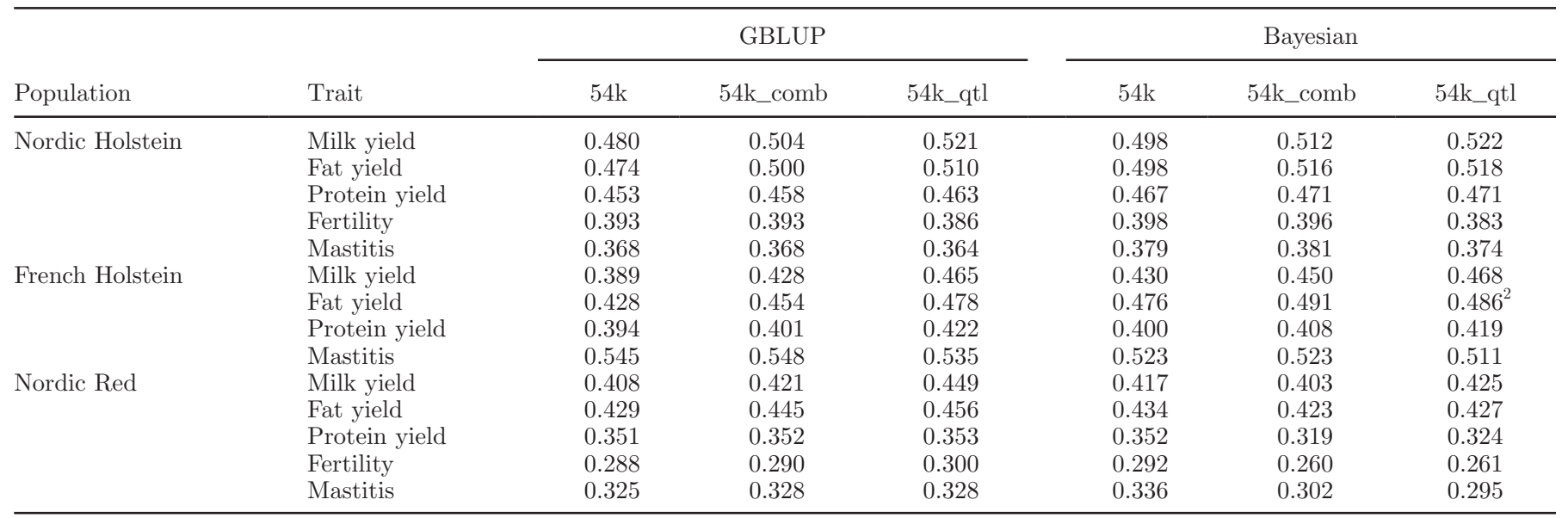

${ }^{1}$ Results in this table are obtained using only markers chosen for the specific trait as QTL markers.

${ }^{2}$ Fixed pi parameter from Nordic Holstein. 
Table A3. Reliabilities of direct genomic values using a genomic BLUP (GBLUP) and a Bayesian 2-mixture model on 54k data, 54k data combined with QTL markers (54k_comb), or 54k data and QTL data treated as separate variance components (54k_qtl)

\begin{tabular}{|c|c|c|c|c|c|c|c|}
\hline \multirow[b]{2}{*}{ Population } & \multirow[b]{2}{*}{ Trait } & \multicolumn{3}{|c|}{ GBLUP } & \multicolumn{3}{|c|}{ Bayesian } \\
\hline & & $54 \mathrm{k}$ & $54 \mathrm{k} \_c o m b$ & $54 \mathrm{k} \_\mathrm{qtl}$ & $54 \mathrm{k}$ & $54 \mathrm{k} \_c o m b$ & $54 \mathrm{k} \_q \mathrm{tl}$ \\
\hline \multirow[t]{5}{*}{ Nordic Holstein } & Milk yield & 0.480 & 0.500 & 0.521 & 0.498 & 0.512 & 0.522 \\
\hline & Fat yield & 0.474 & 0.484 & 0.509 & 0.498 & 0.510 & 0.519 \\
\hline & Protein yield & 0.453 & 0.458 & 0.464 & 0.467 & 0.471 & 0.472 \\
\hline & Fertility & 0.393 & 0.393 & 0.380 & 0.398 & 0.398 & 0.383 \\
\hline & Mastitis & 0.368 & 0.370 & 0.370 & 0.379 & 0.381 & 0.375 \\
\hline \multirow[t]{4}{*}{ French Holstein } & Milk yield & 0.389 & 0.420 & 0.461 & 0.430 & 0.446 & $0.462^{2}$ \\
\hline & Fat yield & 0.428 & 0.437 & 0.481 & 0.476 & 0.491 & 0.489 \\
\hline & Protein yield & 0.394 & 0.401 & 0.417 & 0.400 & 0.406 & 0.419 \\
\hline & Mastitis & 0.545 & 0.545 & 0.520 & 0.523 & 0.520 & 0.517 \\
\hline \multirow[t]{5}{*}{ Nordic Red } & Milk yield & 0.408 & 0.413 & 0.445 & 0.417 & 0.395 & 0.420 \\
\hline & Fat yield & 0.429 & 0.438 & 0.454 & 0.434 & 0.417 & 0.427 \\
\hline & Protein yield & 0.351 & 0.351 & 0.344 & 0.352 & 0.318 & 0.316 \\
\hline & Fertility & 0.288 & 0.290 & 0.298 & 0.292 & 0.258 & 0.261 \\
\hline & Mastitis & 0.325 & 0.327 & 0.324 & 0.336 & 0.298 & 0.285 \\
\hline
\end{tabular}

${ }^{1}$ Results in this table are obtained using only markers chosen for the specific trait and breed as QTL markers.

${ }^{2}$ Fixed pi parameter from Nordic Holstein.

Table A4. Bias of direct genomic values using a genomic BLUP (GBLUP) and Bayesian 2-mixture model on 54k data, 54k data combined with QTL markers (54k_comb), or 54k data and QTL data treated as separate variance components (54k_qtl) ${ }^{1}$

\begin{tabular}{|c|c|c|c|c|c|c|c|}
\hline \multirow[b]{2}{*}{ Population } & \multirow[b]{2}{*}{ Trait } & \multicolumn{3}{|c|}{ GBLUP } & \multicolumn{3}{|c|}{ Bayesian } \\
\hline & & $54 \mathrm{k}$ & $54 \mathrm{k} \_c o m b$ & $54 \mathrm{k} \_q t l$ & $54 \mathrm{k}$ & $54 \mathrm{k} \_c o m b$ & $54 \mathrm{k} \_q t l$ \\
\hline \multirow{5}{*}{ Nordic Holstein } & Milk yield & 0.909 & 0.929 & 0.932 & 0.923 & 0.934 & 0.941 \\
\hline & Fat yield & 0.864 & 0.872 & 0.852 & 0.850 & 0.856 & 0.857 \\
\hline & Protein yield & 0.864 & 0.876 & 0.875 & 0.873 & 0.880 & 0.880 \\
\hline & Fertility & 0.949 & 0.944 & 0.922 & 0.947 & 0.943 & 0.925 \\
\hline & Mastitis & 0.911 & 0.921 & 0.925 & 0.921 & 0.930 & 0.929 \\
\hline \multirow[t]{4}{*}{ French Holstein } & Milk yield & 0.758 & 0.803 & 0.821 & 0.818 & 0.839 & 0.850 \\
\hline & Fat yield & 0.855 & 0.853 & 0.844 & 0.863 & 0.861 & $0.863^{2}$ \\
\hline & Protein yield & 0.705 & 0.719 & 0.726 & 0.716 & 0.724 & 0.729 \\
\hline & Mastitis & 0.779 & 0.783 & 0.774 & 0.768 & 0.770 & 0.743 \\
\hline \multirow[t]{5}{*}{ Nordic Red } & Milk yield & 0.876 & 0.877 & 0.857 & 0.869 & 0.866 & 0.859 \\
\hline & Fat yield & 0.835 & 0.835 & 0.805 & 0.804 & 0.810 & 0.812 \\
\hline & Protein yield & 0.781 & 0.784 & 0.774 & 0.775 & 0.776 & 0.770 \\
\hline & Fertility & 0.988 & 0.991 & 0.987 & 0.973 & 0.975 & 0.965 \\
\hline & Mastitis & 0.918 & 0.919 & 0.905 & 0.884 & 0.891 & 0.889 \\
\hline
\end{tabular}

${ }^{1}$ Results in this table are obtained using the entire marker set from the linkage disequilibrium-chip as QTL markers.

${ }^{2}$ Fixed pi parameter from Nordic Holstein.

Table A5. Variance of total direct genomic values (DGV) and partial DGV for either 54k or QTL data for validation animals in the scenario with separate variance components

\begin{tabular}{|c|c|c|c|c|c|c|c|}
\hline \multirow[b]{2}{*}{ Population } & \multirow[b]{2}{*}{ Trait } & \multicolumn{3}{|c|}{ GBLUP } & \multicolumn{3}{|c|}{ Bayesian } \\
\hline & & $54 \mathrm{k}$ & QTL & Total & $54 \mathrm{k}$ & QTL & Total \\
\hline \multirow[t]{5}{*}{ Nordic Holstein } & Milk yield & 30.6 & 17.4 & 58.6 & 32.3 & 16.4 & 57.6 \\
\hline & Fat yield & 22.7 & 25.0 & 56.1 & 26.5 & 25.3 & 56.8 \\
\hline & Protein yield & 31.1 & 9.9 & 51.0 & 32.2 & 10.8 & 51.0 \\
\hline & Fertility & 45.9 & 12.2 & 73.6 & 46.7 & 12.1 & 74.1 \\
\hline & Mastitis & 26.0 & 8.1 & 43.1 & 27.3 & 8.2 & 43.1 \\
\hline \multirow[t]{4}{*}{ French Holstein } & Milk yield & 45.8 & 16.2 & 72.1 & 51.0 & 12.9 & 69.1 \\
\hline & Fat yield & 40.9 & 18.5 & 72.6 & 50.6 & 19.0 & 74.8 \\
\hline & Protein yield & 58.4 & 5.7 & 76.0 & 61.2 & 5.1 & 75.7 \\
\hline & Mastitis & 52.5 & 11.4 & 78.9 & 53.1 & 15.1 & 80.2 \\
\hline \multirow[t]{5}{*}{ Nordic Red } & Milk yield & 25.7 & 15.6 & 50.0 & 26.5 & 16.6 & 51.3 \\
\hline & Fat yield & 20.9 & 16.7 & 48.0 & 21.7 & 16.4 & 48.7 \\
\hline & Protein yield & 30.0 & 10.0 & 49.0 & 31.0 & 11.0 & 50.7 \\
\hline & Fertility & 37.9 & 8.1 & 57.9 & 39.4 & 12.1 & 60.4 \\
\hline & Mastitis & 27.7 & 7.1 & 41.6 & 29.9 & 8.5 & 42.7 \\
\hline
\end{tabular}

